\title{
El Arte de Curar: estudio sobre Vías de Administración. Diferencias entre medio rural y urbano
}

\author{
Jaime González Gonzáleza, Alberto Berrocoso Martínez ${ }^{\mathrm{b}}$, Leonor de Arriba Muñoz ${ }^{\mathrm{b}}$ \\ Guillermo Rico García ${ }^{a}$, Ana Izaguirre Zapateraa ${ }^{a} M^{a}$ Jesús Alfonso Izquierdoa
}

\begin{abstract}
${ }^{a}$ Centro de Salud de Navamorcuende (Toledo).

${ }^{b}$ Centro de Salud de Santa Maria de Benquerencia (Toledo).
\end{abstract}

Correspondencia: Jaime González González, Centro de Salud Navamorcuende, $\mathrm{C} /$ Palacio s/n, 45630 - Navamorcuende (Toledo, España). Telf.: 925868400, e-mail: jaimeg@sescam.jccm.es.

Recibido el 17 de marzo de 2009.

Aceptado para su publicación el 22 de mayo de 2009.

\section{RESUMEN}

Objetivo. Conocer las preferencias de vías de administración de medicamentos de los pacientes y las diferencias según sea medio urbano o medio rural.

Diseño. Estudio observacional, descriptivo, transversal.

Participantes. Pacientes de atención primaria cuyo motivo de consulta fue dolor y éste precisó tratamiento analgésico.

Mediciones principales. Escala visual del dolor para la variable intensidad del dolor. Otras variables: sociodemográficas (sexo, edad, lugar de residencia y nivel de estudios), preferencias de vía de administración de tratamiento y eficacia de vía de administración.

Resultados. Se realizaron 400 encuestas (33\% hombres y $66 \%$ mujeres, con edad media de 58 años). Habitaban en ciudad un $59 \%$ y un $41 \%$ en medio rural. El $44.6 \%$ calificó su dolor como moderado, el $42 \%$ como intenso, el $4.2 \%$ leve y el $4.2 \%$ insoportable. El $48 \%$ prefería tratamiento parenteral, el $50 \%$ oral y el $1.8 \%$ tratamiento tópico. Preguntados sobre eficacia a igual dosis, el $74 \%$ se inclinó por tratamiento parenteral, el $8.9 \%$ por tratamiento oral y el $19.6 \%$ igual.

Las personas mas jóvenes y con estudios universitarios prefieren el tratamiento oral. En el medio rural la preferencia por los tratamientos parenterales es mayor que en el medio urbano, aunque la diferencia no es estadísticamente significativa.

Conclusiones. Las preferencias por los tratamientos parenterales son superiores en el medio rural y en las personas mayores de 65 años, debido principalmente a que creen que es más potente. Los jóvenes, los que poseen estudios universitarios y los que residen en el medio urbano prefieren tratamiento oral, principalmente por ser más cómodo. La vía de administración del medicamento debe ser consensuado con el paciente, puesto que la predisposición de éste puede ayudar al efecto terapéutico de dicho medicamento.

Palabras clave. Prescripción de Medicamentos. Infusiones Parenterales. Administración Oral.

\section{ABSTRACT}

The art of curing: Difference in Administration routes in rural and urban settings

Objective. To determine patient preference as regards administration route and the differences between urban and rural settings.

Design. Observational, descriptive, cross-sectional study

Participants. Patients who visited their primary care doctor due to pain and who needed analgesic treatment.

Principal measurements. Visual pain scale for the pain intensity variable. Other variables: sociodemographic (sex, age, place of residence, educational level), administration route preference and administration route efficacy.

Results. A total of 400 subjects (33\% men and $66 \%$ women with a mean age of 58 years) were surveyed. $59 \%$ lived in an urban setting and $41 \%$ in a rural setting. $44.6 \%$ classified their pain as moderate, $42 \%$ as severe, $4.2 \%$ as mild and $4.2 \%$ as unbearable. $48 \%$ preferred parenteral, treatment, $50 \%$ oral treatment and $1.8 \%$ topical treatment. When asked about the efficacy at equal doses, $74 \%$ preferred parenteral treatment, $8.9 \%$ oral treatment and $19.6 \%$ thought it was the same for both.

Younger persons with a university education preferred oral treatment. Preference for parenteral route was higher in the rural setting than in the urban setting. although the difference was not statistically significant.

Conclusions. Preference for parenteral administration is greater in the rural setting and in persons over 65 years, mainly because they consider this route more effective. Younger persons, those with a university education and those who live in an urban setting prefer oral treatment, mainly because it is more convenient. The administration route should be decided on with the patient because the patient's willingness to be treated may assist in the therapeutic effect of the medication.

Key words: Prescriptions, Drug. Infusions, Parenteral. Administration, Oral.

Estudio presentado en el IX Congreso de Atención Primaria de Castilla-La Mancha (Guadalajara, 5-7 de junio de 2008). 


\section{INTRODUCCIÓN}

Una de los motivos de visita más frecuentes en la consulta diaria en atención primaria es el tratamiento del dolor ${ }^{1}$, por lo que en este nivel de atención debemos estar preparados para resolver estas demandas. No solamente debemos manejar perfectamente la escalera terapéutica de la $\mathrm{OMS}^{2}$ para el control del dolor, pues en muchas ocasiones aparecen otros factores que no son puramente la relación dolor-dosis de medicamento, factores muy importantes a la hora de poner un tratamiento, lo cual debe tenerse muy en cuenta por parte de los médicos en la consulta.

En nuestras consultas, además de administrar el medicamento adecuado, debemos intentar que el paciente tenga confianza en que se va a curar y en que la solución que le proponemos para su problema es la más adecuada.

Los factores biopsicosociales del paciente, el ambiente, el tono de voz, el leguaje, tanto el verbal como el gestual, van a condicionar la empatía medico-paciente $^{3}$, la cual debe ser un factor a nuestro favor a la hora de la consulta de atención primaria, en la que conocemos perfectamente a nuestros pacientes, sus problemas, e incluso su entorno.

Curar no solamente es prescribir un medicamento apropiado ante un dolor por el que consulta el paciente, sino que hay que intentar hacerlo de forma consensuada con él para que surja el efecto esperado, dada la predisposición del paciente ante una solución a su problema.

Por otro lado, la población que acude a nuestras consultas de atención primaria es muy variada, existiendo diferencias muy marcadas entre la población rural y la población urbana ${ }^{4}$, tanto en edad ${ }^{5}$ como en presentación de enfermedades (artrosis, obesidad, etc.), hábitos dietéticos y ejercicio físico.

Para ver la percepción subjetiva de nuestros pacientes ante la prescripción de medicamentos y la variabilidad en la vía de administración, según su lugar de residencia y otras características biopsicosociales, presentamos este trabajo, en el cual analizamos las diferencias existentes entre distintos grupos, prestando un especial interés a las diferencias existentes entre las poblaciones de medio rural y medio urbano.

El objetivo del estudio fue valorar la confianza de los pacientes en las vías de administración de los medicamentos, mostrando las diferencias entre grupos según edad, sexo, lugar de residencia (medio urbano o rural), intensidad del dolor y nivel de estudios.

\section{MATERIAL Y MÉTODO}

Para llevar a cabo este estudio transversal, hemos realizado 400 encuestas a pacientes mayores de 14 años (criterio de exclusión: edad inferior a 14 años) que consultaron por dolor, y que precisaron tratamien-

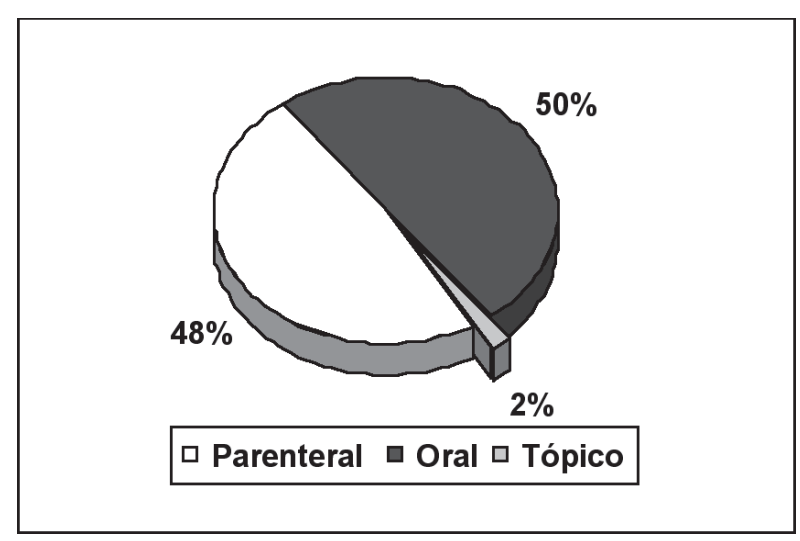

Figura 1. Preferencias de tratamiento.

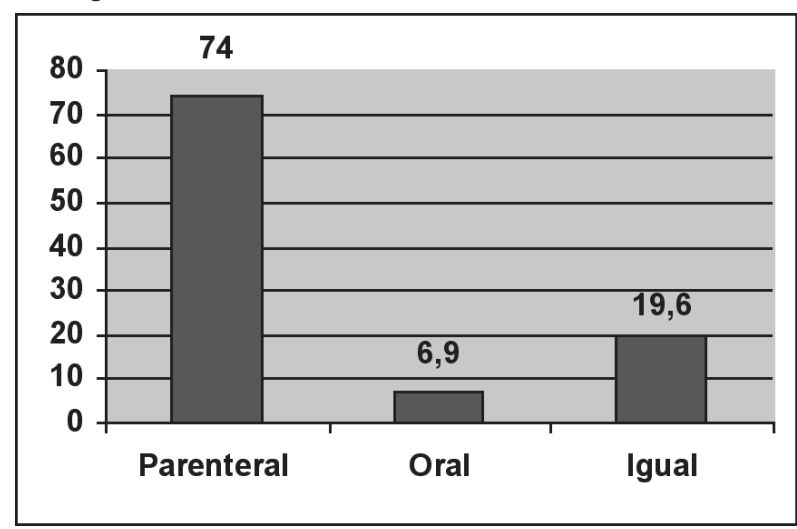

Figura 1. ¿Cuál cree que es el tratamiento más eficaz?

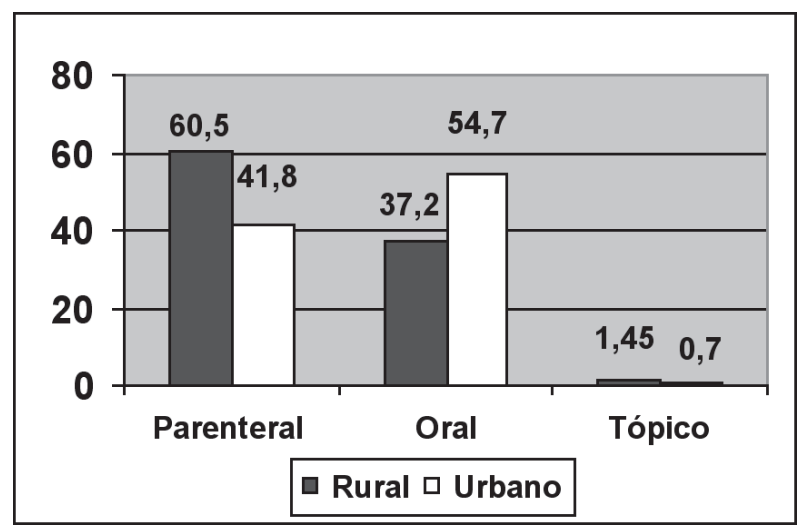

Figura 1. Preferencias de tratamiento.

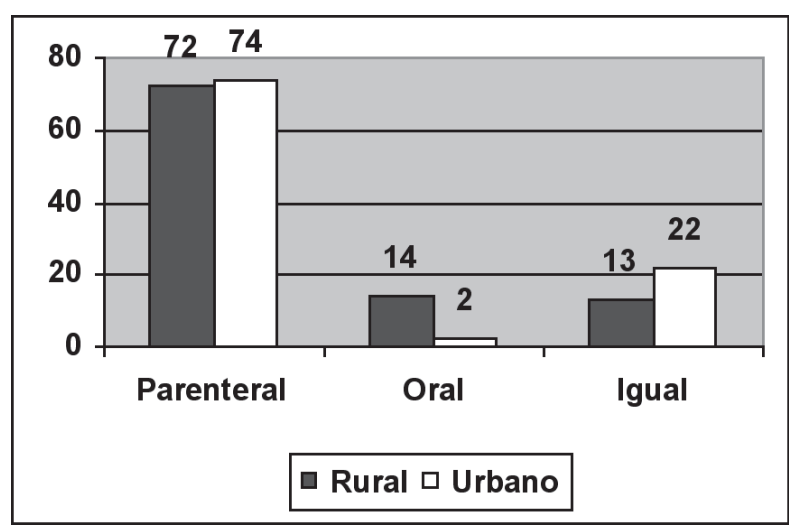

Figura 1. Recepción de eficacia 
to analgésico. Los datos fueron obtenidos, durante el periodo comprendido entre el 1 de octubre de 2008 y el 30 de abril de 2009, en las consultas urbanas del Centro de Salud de Santa Maria de Benquerencia y en los siete consultorios rurales del Centro de Salud de Navamorcuende. Las variables estudiadas fueron: edad, sexo, nivel de estudios, intensidad del dolor, consultas anteriores por dolor, preferencias sobre vías de administración del tratamiento para el dolor, y motivos de las mismas, y consideración de los pacientes sobre efectividad de las distintas vías de administración a igualdad de dosis.

\section{RESULTADOS}

Se realizaron 400 encuestas de las cuales el 33\% correspondieron a hombres y el $66 \%$ a mujeres, con una edad media de 58 años de edad. El 50\% tenían estudios primarios, el $25 \%$ no tenían estudios, el $8.9 \%$ estudios medios y el $16 \%$ estudios universitarios. Habitaban en ciudad el $59 \%$ y el $41 \%$ en el medio rural. El $44.6 \%$ calificó su dolor como moderado y el $42 \%$ como intenso (solamente el $4.2 \%$ lo calificó como leve y sólo el $4.2 \%$ como insoportable). El $66.1 \%$ había consultado anteriormente por su dolor.

Respecto al tratamiento para el dolor, el 48\% manifestó su preferencia por la vía de administración parenteral y el 50\% por la vía oral (solamente el 1.8\% prefirió tratamiento tópico) (figura 1).

Preguntados por el motivo de esta decisión, las personas que prefirieron tratamiento oral afirmaron mayoritariamente que el motivo de su elección era la comodidad de la vía oral (82\%), sin embargo, los pacientes que prefirieron el tratamiento parenteral declararon que la razón de esta elección era la "mayor potencia" del tratamiento.

Por último, preguntados sobre cuál creían que era el tratamiento mas eficaz a igual dosis, el $74 \%$ se manifestó a favor del tratamiento parenteral, el $8.9 \%$ a favor de la vía oral y el $19.6 \%$ opinó que eran similares.

Analizando las diferencias entre grupos mediante el programa estadístico SPSS 14, se observó que las personas mas jóvenes (menores de 40 años) prefieren con más frecuencia el tratamiento oral respecto a las personas mayores de 65 años, las cuales prefieren más frecuentemente el tratamiento parenteral. Las personas con estudios universitarios prefieren el tratamiento oral y son más sensibles a la igualdad de eficacia ante igualdad de dosis.

Entre personas de ámbito urbano y rural encontramos algunas diferencias, aunque no estadísticamente significativas $(P>0.05)$, observando que las personas que viven en el medio rural prefieren con más frecuencia tratamientos parenterales, mientras que esta proporción es inferior en las que pertenecen al medio urbano. Sin embargo, tanto en el medio rural como en el medio urbano, los entrevistados consideran más eficaz el tratamiento parenteral respecto al tratamiento oral a igualdad de dosis

\section{DISCUSIÓN}

En pleno siglo XXI, en el que la ciencia avanza a pasos insospechados, se habla de terapias genéticas o de medicamentos biológicos, y cada día aparecen nuevos medicamentos y nuevas formas de presentación (distintos medicamentos en un solo comprimido, unidosis diaria, semanal o incluso mensual), suponiendo menos problemas para el paciente a la hora de tomar los medicamentos. A pesar de ello, corroboramos con nuestro trabajo que aún en nuestros tiempos esos dichos populares, como "la gripe se pasa con unas buenas inyecciones de esas que duelen" o "el lumbago se pasa con una caja de inyecciones", siguen muy en boca de nuestros pacientes, sobre todo en los de mayor edad, en los de un nivel cultural inferior y en los que residen en el medio rural, aunque no de forma exclusiva.

Con nuestro trabajo queremos hacer hincapié en que el paciente puede y debe contribuir a la hora de poner tratamiento a su enfermedad y que la predisposición del paciente ante una enfermedad es un arma a nuestro favor a la hora de instaurar tratamiento. Cuando un paciente nos consulta por un dolor y nos dice que quiere una caja de inyecciones, siempre y cuando sea posible médicamente, debemos tener en cuenta su decisión, pues ese paciente va a estar predispuesto a curarse con esa medicación y, aunque solamente sea por el efecto placebo o pseudoplacebo, debemos intentar dar respuesta al paciente tal y como él desea. No queremos plantear que siempre haya que hacer lo que dice el paciente, pero creemos que es importante.

Ante un paciente que consulta por lumbago y nos dice que con una caja de inyecciones se le pasa, si nos empeñamos en pautarle AINEs orales, en muchas ocasiones sólo conseguimos que no tome la medicación y que en dos o tres días acuda de nuevo a la consulta, o a urgencias, hasta conseguir su objetivo, además de generar un mayor gasto sanitario.

La pregunta “¿qué quiere usted?”, siempre y cuando sea posible y factible médicamente, debe ser respondida con un "de acuerdo", pues eso nos ayudará mucho en la relación medico-paciente y en solventar los problemas diarios de la consulta.

Como conclusiones:

1- Existen diferencias entre los pacientes según su edad, lugar de residencia, nivel de estudios y sexo sobre la percepción del tratamiento que prefieren para su dolor.

2- Las personas que pertenecen al medio rural y las que son mayores de 65 años prefieren tratamientos parenterales para el dolor, debido principalmente a que creen que son más potentes que por vía oral 
3- Las personas jóvenes, con estudios universitarios y pertenecientes al medio urbano prefieren tratamiento oral para sus dolencias, principalmente por ser más cómoda su administración.

4- La vía de administración del medicamento debe ser consensuada con el paciente, puesto que la predisposición de éste puede favorecer el efecto terapéutico de dicho medicamento.

\section{BIBLIOGRAFÍA}

1. Gil Gregorio P, Moreno A, Rodríguez MA, Zarco J (en representación de los investigadores del estudio ADA). Manejo del dolor del anciano en Atención Primaria (estudio ADA). Rev Clin Esp 2007; 207:166-71.

2. Dulce Tello S, Rollán Arribas B, Lopez Martinez E, Camarasa Perez J. Terapéutica en Atención Primaria. Uso de los opioides en pacientes con dolor oncológico. Semergen 2007; 33:520-8.

3. Garcia-Morillo S, Bernabeu-Wittel M, Cassani M, Rincon M, Yerro Páez V, Ollero Barturone M. Influencia de los factores biopsicosociales en el grado de empatía de la relación médico-en- fermo en una cohorte de pluripatológicos. Rev Clin Esp 2007; 207:379-82.

4. Martin Lesende I, Garcia Rodríguez A, Abajo Angulo JL, Olabarria Ateca V, Gonzalez Garcia J, Rueda Alonso E et al. Comparación de la situación sanitaria en base a una Valoración Geriátrica Integral, entre una población rural y urbana de 75 o más años de una misma zona de salud. Rev Esp Geriatr Gerontol 2001; 36:150-5.

5. Simunovi_R, Kati_M, Todorovi_ G, Vinter-Repalust N, Petric D. Differences between rural and urban general practice activities. Acta Med Croatia 2007; 61:13-8.

6. Mclean G, Guthrie B, Sutton M. Differences in the quality of primary medical care services by remoteness from urban settlementes. Qual Saf Health care 2007; 16:446-9.

7. Ripoll Lozano MA, Jiménez Arce JI, Pedraza Dueñas A. Variablity in the antibiotics prescription in the Avila Province. Rev Esp Quimioter 2007; 20: 40-50.

8. Britt H, Miles DA, Bridges-Webb C, Neary S, Charles J, Traynor V. A comparison of country and metropolitan general practice. Med J Aust 1993; 159(Suppl):S9-64.

9. Vlastos IM, Mpatistakis AG, Glouskou KK. Health needs in rural areas and the efficacy and cost-effectiveness of doctors and nurses. Aust J Rural Health 2005; 13:359-63. 